\title{
The tip of the iceberg: cardiac magnetic resonance imaging findings in patients with myocardial infarction with non-obstructive coronary arteries: preliminary data from the Polish single-centre registry
}

\author{
Piotr Gościniak ${ }^{1}$, Tomasz Baron², Robert Józwa ${ }^{3}$, Małgorzata Pyda ${ }^{4}$ \\ 'Laboratory for Non-Invasive Cardiac Investigations, Tomasz Sokolowski Public Hospital, Pomeranian Medical University, Szczecin, Poland \\ ¿Uppsala Clinical Research Centre and Department of Medical Sciences, Cardiology, Uppsala University, Uppsala, Sweden \\ ${ }^{3}$ Department of Cardiology, Maria Curie-Skłodowska Regional Hospital, Szczecin, Poland \\ ${ }^{4}$ Cardiac Magnetic Resonance Laboratory, $1^{\text {st }}$ Department of Cardiology, Poznan University of Medical Sciences, Poznan, Poland
}

\section{INTRODUCTION}

The diagnosis of myocardial infarction with non-obstructive coronary arteries (MINOCA) is possible after performing coronary angiography in patients with a clinical presentation suggesting myocardial infarction (MI) if the criteria of an acute coronary syndrome (ACS) according to the European Society of Cardiology (ESC) universal definition of MI [1] are met and no significant obstructive coronary lesions are found (i.e. no $\geq 50 \%$ stenosis in any of the potential infarct-related arteries), with no other clinically evident cause of the acute clinical presentation [2].

Because cardiac troponin is a nonspecific biomarker, the diagnosis of MINOCA requires additional diagnostic tests. It is necessary to exclude most typical causes of myocardial injury, such as myocarditis or stress-induced cardiomyopathy, which are not included in the $\mathrm{Ml}$ category. A major role in the diagnostic evaluation is played by cardiac magnetic resonance (CMR) imaging, which is currently the only available method that allows a non-invasive evaluation of myocardial tissue characteristics. CMR allows the distinction between ischaemic and non-ischaemic myocardial injury, evaluation of the presence of myocardial oedema, fibrosis, or scar, and, with new imaging sequences (mapping), quantitative evaluation of oedema or inflammation.

Until now, only one Polish centre has reported their experience with this clinical entity [3].
The aim of the study was to evaluate the incidence of MINOCA in the population of West Pomerania, Poland and the utility of CMR in these patients based on 12-month follow-up in a large cardiology unit. The study employed a prospective observational registry design.

\section{METHODS}

From January to December 2017, a total of 5006 patients were hospitalised in our unit. During that period, 2756 coronary angiographies were performed, including 604 procedures in the setting of ACS (327 in patients with non-ST-segment elevation MI [NSTEMI] and 277 in patients with ST-segment elevation MI [STEMI]). Patients with unstable angina were not included in the study. Among these 604 patients, 83 (13.7\%) persons had no significant obstructive coronary lesions. Patients with confirmed pulmonary embolism $(n=11)$, previous $M I(n=17)$, aortic dissection $(n=1)$, severe valvular heart disease $(n=10)$, known cardiomyopathy $(n=8)$, severe renal impairment $(n=8)$, and other contraindications to CMR $(n=7)$ were also excluded from the study.

Ultimately, we analysed 21 (3.5\%) patients, including nine women and 12 men with an ACS, who were found to have no significant obstructive coronary lesions, an abnormal troponin level, and no other clinically evident cause of the acute clinical presentation. These were the only patients initially diagnosed with MINOCA in our study population. The mean patient age was 46 years (range $27-74$ years).

\section{Address for correspondence:}

Piotr Gościniak, MD, PhD, Laboratory for Non-Invasive Cardiac Investigations, Tomasz Sokolowski Public Hospital, Pomeranian Medical University, Unii Lubelskiej 1 , 71-252 Szczecin, Poland, tel: 509246346, e-mail: piotr.gosciniak@life.pl

Received: 25.11.2018 Accepted: 2.01.2019 Available as AoP: 2.01.2019

Kardiologia Polska Copyright (c) Polish Cardiac Society 2019 
Table 1. Clinical characteristics of patients with myocardial infarction with non-obstructive coronary arteries (MINOCA)

\begin{tabular}{|c|c|c|c|c|c|}
\hline & $\begin{array}{c}\text { Total } \\
(n=21 *)\end{array}$ & $\begin{array}{l}\text { Group 1: } \\
\text { true MINOCA } \\
\quad(n=8)\end{array}$ & $\begin{array}{l}\text { Group 2: } \\
\text { myocarditis } \\
(n=8)\end{array}$ & $\begin{array}{l}\text { Group 3: takotsubo } \\
\text { cardiomyopathy } \\
(n=4)\end{array}$ & $\mathbf{p}$ \\
\hline Women & 42.9 & 62.5 & 12.5 & 100 & 0.0898 \\
\hline Age [years] & $46 \pm 14$ & $48 \pm 14$ & $37 \pm 7$ & $62 \pm 17$ & $\begin{array}{c}0.0132 \\
\text { G2 vs. G3: } 0.0106\end{array}$ \\
\hline \multicolumn{6}{|l|}{ Risk factors: } \\
\hline Current smoking & 25.0 & 42.9 & 25.0 & 0 & 0.2776 \\
\hline Diabetes & 4.8 & 0 & 0 & 25 & 0.2000 \\
\hline Hypertension & 35.0 & 37.5 & 28.6 & 50 & 0.8444 \\
\hline Dyslipidaemia & 14.3 & 12.5 & 0 & 50 & 0.0877 \\
\hline History of HF & 0 & 0 & 0 & 0 & \\
\hline History of MI & 0 & 0 & 0 & 0 & \\
\hline History of stroke & 0 & 0 & 0 & 0 & \\
\hline COPD & 10.5 & 12.5 & 0 & 25 & \\
\hline History of thromboembolism & 0 & 0 & 0 & 0 & \\
\hline Peripheral vascular disease & 0 & 0 & 0 & 0 & \\
\hline Thyroid disease & 4.8 & 12.5 & 0 & 0 & \\
\hline \multicolumn{6}{|l|}{ Pharmacotherapy on admission: } \\
\hline ASA & 4.8 & 0 & 0 & 25 & \\
\hline VKA/NOAC & 0 & 0 & 0 & 0 & \\
\hline$\beta$-blocker & 14.4 & 12.5 & 12.5 & 25 & \\
\hline ACEI & 24 & 37.5 & 12.5 & 25 & \\
\hline Statin & 9.6 & 12.5 & 0 & 25 & \\
\hline \multicolumn{6}{|l|}{ Pharmacotherapy at discharge: } \\
\hline DAPT & 48 & 100 & 0 & 50 & \\
\hline ASA & 9.6 & 0 & 25 & 25 & \\
\hline$\beta$-blocker & 67.2 & 100 & 25 & 100 & \\
\hline ACEI & 86.4 & & 75 & 100 & \\
\hline Statin & 67.2 & 100 & 25 & 100 & \\
\hline \multicolumn{6}{|l|}{ Electrocardiogram findings: } \\
\hline ST elevation & 33 & 37.5 & 50 & 0 & 0.3932 \\
\hline ST depression & 43 & 32 & 25 & 0 & \\
\hline T wave abnormalities & 23 & 31 & 12.5 & 100 & \\
\hline \multicolumn{6}{|l|}{ Laboratory findings: } \\
\hline Troponin T [ng/L] & $473(203-836)$ & $470.5(244.5-1013)$ & $563(165.5-928.5)$ & 416.5 (283.7-726.5) & 0.9529 \\
\hline \multirow[t]{4}{*}{ CRP $[\mathrm{mg} / \mathrm{L}]$} & $5.7(2.5-59)$ & $2.6(1.4-5.1)$ & $61(40.6-72.5)$ & $8.8(5.1-51.4)$ & 0.0127 \\
\hline & & & & & G1 vs. G2: 0.0074 \\
\hline & & & & & G1 vs. G3: 0.0745 \\
\hline & & & & & G2 vs. G3: 0.3502 \\
\hline Creatinine [mg/dL] & $0.87(0.74-1.01)$ & $0.78(0.66-0.94)$ & $0.89(0.77-1.06)$ & $0.83(0.71-1.12)$ & 0.3629 \\
\hline NT-proBNP [pg/mL] & 1456 (159-3189) & $1456(146-3669)$ & 747 (109-857.0) & $2936(1136-5369)$ & 0.1975 \\
\hline WBC $\left[10^{3} / \mu \mathrm{L}\right]$ & $9.4(7.4-10.7)$ & $7.9(6.8-9.7)$ & $9.5(8.5-12.5)$ & $9.6(6.9-11.1)$ & 0.3568 \\
\hline \multirow[t]{4}{*}{ Haemoglobin [g/dL] } & $13.9(13.3-14.5)$ & $13.8(12.3-14.4)$ & $14.6(14-16.0)$ & $13.3(12.7-13.4)$ & 0.0129 \\
\hline & & & & & G1 vs. G2: 0.0403 \\
\hline & & & & & G1 vs. G3: 0.3949 \\
\hline & & & & & G2 vs. G3: 0.0085 \\
\hline
\end{tabular}


Table 1 (cont.). Clinical characteristics of patients with myocardial infarction with non-obstructive coronary arteries (MINOCA)

\begin{tabular}{lccccc} 
& $\begin{array}{c}\text { Total } \\
(\mathbf{n}=\mathbf{2 1 *})\end{array}$ & $\begin{array}{c}\text { Group 1: } \\
\text { true MINOCA } \\
(\mathbf{n}=\mathbf{8})\end{array}$ & $\begin{array}{c}\text { Group 2: } \\
\text { myocarditis } \\
(\mathbf{n}=\mathbf{8})\end{array}$ & $\begin{array}{c}\text { Group 3: takotsubo } \\
\text { cardiomyopathy } \\
(\mathbf{n}=\mathbf{4})\end{array}$ \\
\hline $\begin{array}{l}\text { CMR examination findings: } \\
\text { Time to CMR [days] }\end{array}$ & $5(3-7)$ & $4,5(3.5-5.5)$ & $5(3-6.5)$ & $7(5.5-7)$ & 0.2249 \\
LVEDV [mL] & $149 \pm 52$ & $141 \pm 34$ & $146 \pm 20$ & $127 \pm 45$ & 0.6270 \\
LVESV [mL] & $69 \pm 52$ & $63 \pm 24$ & $60 \pm 14$ & $49 \pm 21$ & 0.5228 \\
LVEF [\%] & $56 \pm 13$ & $57 \pm 9$ & $59 \pm 6$ & $62 \pm 13$ & 0.7756 \\
LVM [g] & $124 \pm 34$ & $116 \pm 29$ & $127 \pm 19$ & $111 \pm 22$ & 0.5375 \\
Oedema (T2-weighted images) & 28.5 & 25 & 50 & 0 & 0.2198 \\
LGE ischaemic pattern & 33.3 & 87.5 & 0 & 0 & $<0.005$ \\
LGE non-ischaemic pattern & 42.9 & 0 & 100 & 0 & $<$ \\
No LGE & 23.8 & 12.5 & 0 & 0.0001 \\
\hline
\end{tabular}

Categorical variables are presented as percentage. Normally distributed continuous variables are presented as mean \pm standard deviation. Non-normally distributed continuous variables are presented as median and interquartile range.

*One patient with no changes in CMR. ACEI — angiotensin-converting enzyme inhibitor; ASA — acetylsalicylic acid; CMR — cardiac magnetic resonance; COPD — chronic obstructive pulmonary disease; CRP — C-reactive protein; DAPT — dual antiplatelet therapy; HF — heart failure; LGE - late gadolinium enhancement; LVM — left ventricular mass; LVEDV — left ventricular end-diastolic volume; LVEF — left ventricular ejection fraction; LVESV — left ventricular end-systolic volume; $\mathrm{Ml}$ - myocardial infarction; NOAC — non-vitamin K antagonist oral anticoagulant; NT-proBNP — N-terminal pro-B-type natriuretic peptide; VKA — vitamin K antagonist; WBC — white blood cells

All patients were admitted with a clinical presentation suggesting MI and met the criteria set forth in the ESC universal definition of MI [2]. On admission, NSTEMI was diagnosed in $14(66 \%)$ patients, and STEMI was diagnosed in seven (33\%) patients. Normal coronary arteries were defined as non-obstructive lesions and stenoses up to $50 \%$. Following coronary angiography, the patients were initially diagnosed with MINOCA. The final diagnosis was based on echocardiography, CMR, and laboratory tests.

Cardiac magnetic resonance was performed in the entire study group ( $\mathrm{n}=21$ ) within seven days of admission using a 1.5T system (Avanto, Siemens, Erlangen, Germany) and included spin echo, turbo spin echo, and gradient recalled echo sequences in T1- and T2-weighted images before and after administration of a contrast agent (Gadovist, Bayer AG, Leverkusen, Germany). No adverse effects were observed. CMR images were interpreted by two experts in cardiology and radiology. The diagnosis of myocarditis was based on the Lake Louise Consensus Criteria [4]. The diagnostic criteria for the diagnosis of takotsubo cardiomyopathy were based on the Mayo Clinic recommendations [5]. The presence and location of late gadolinium enhancement (LGE) were used to distinguish between ischaemic and non-ischaemic aetiology of myocardial injury.

\section{Statistical analysis}

Normally distributed continuous variables were presented as mean \pm standard deviation. One-way analysis of variance (ANOVA) was used to determine the significance of differences between the mean values, and a post-hoc Tukey test was used for pairwise comparisons. Continuous variables with a non-normal distribution were presented as median and interquartile range (IQR). A nonparametric Mann-Whitney $U$ test was used to determine the significance of differences between distributions. Categorical variables were presented as the number of patients and frequency and compared with the $\chi^{2}$ test. For all analyses, two-sided $p$-values $<0.05$ were considered significant. Statistical analyses were performed using the SAS Software version 9.2. (SAS Institute, Cary, NC, USA).

\section{RESULTS AND DISCUSSION}

Troponin T level was significantly elevated in all patients (upper reference limit $14 \mathrm{ng} / \mathrm{L}$, median $473.0 \mathrm{ng} / \mathrm{L}$; IQR 186.5-905). Other laboratory parameters are shown in Table 1. In one patient, optical coherence tomography (OCT) was performed during coronary angiography to identify intramural lesions (plaque rupture, dissection).

The median time from admission to CMR was five days (IQR 3-7 days). In the study group $(\mathrm{n}=21)$, the diagnosis of $\mathrm{MI}$ was confirmed based on CMR imaging in eight (38\%) patients (including five patients with NSTEMI and three patients with STEMI; mean age of 48 years). Myocarditis was diagnosed in eight (38\%) patients (including four patients with NSTEMI and four patients with STEMI; mean age of 37 years). Stress-induced cardiomyopathy was diagnosed in four (19\%) patients (only women, at a mean age of 62 years). In one patient, CMR imaging yielded a normal result. 
In our study, an initial diagnosis of MINOCA was made in 21 (3.5\%) of 604 patients admitted due to an ACS, more commonly in men. A minority of these patients had risk factors for cardiovascular disease (Table 1). In the subgroup with ischaemic LGE confirmed by CMR (true MINOCA), women were more prevalent. In the subgroup with myocarditis, higher troponin levels and elevated inflammation markers were observed more commonly, and this group included the youngest patients with myocardial injury in our study (Table 1). These observations are consistent with the available literature data [2].

Unfortunately, CMR does not identify the pathomechanism of ischaemia, such as plaque rupture, erosion or ulceration, or coronary artery dissection. For the latter purpose, additional intracoronary imaging methods, such as intravascular ultrasound (IVUS) or OCT, are recommended because they allow a detailed evaluation of vessel wall anatomy. In our study, we used OCT for detailed evaluation of coronary arteries in one patient in whom spontaneous artery dissection was ultimately diagnosed and a drug-eluting stent was implanted. In that patient, CMR imaging showed no LGE. The rate of coronary artery wall disruption or dissection is not well known, and thus more frequent use of intracoronary imaging has been postulated. In the study by Ouldzein et al. [6], IVUS showed plaque rupture or ulceration in about $40 \%$ of patients with MINOCA.

The main strength of our analysis is the demonstration that early CMR imaging in this specific patient subset allowed proper and accurate diagnosis to be made before discharge in all patients. This led to immediate initiation of appropriate treatment in these particularly challenging patients. We believe that our findings confirm the role of CMR in early cardiac imaging in patients with a presumed diagnosis of MINOCA [7].

Several limitations of our study should be taken into account. To reduce the selection bias, we studied a prospectively defined patient group that fulfilled strict inclusion criteria comprising the clinical presentation of MI with elevated troponin level, no significant obstructive coronary lesions, and no other clinically evident cause of the acute clinical presentation. We excluded patients with normal electrocardiogram, and thus all patients in our study had ST-T changes. The exclusion criteria also comprised not performing CMR during the hospital stay. These factors probably contributed to the relatively low number of patients in our study. CMR findings were analysed collectively by an experienced radiologist and a certified cardiologist who previously participated in national Polish registries, and variation in their interpretations of CMR findings was not evaluated. OCT was performed in only one patient because this method was not available on a $24 / 7$ basis.

Although the prognosis in patients with MINOCA is better than in those with a true $\mathrm{MI}$, it is worse compared to the general population. A higher rate of cardiovascular events may be related to inappropriate diagnosis. However, prior to the preparation of the present manuscript, major cardiovascular adverse events including all-cause mortality, rehospitalisation for $\mathrm{MI}$, ischaemic stroke, and heart failure were not recorded in the overall study group. Patients with confirmed true MINOCA received dual antiplatelet therapy for 12 months along with a statin.

In our study, early use of CMR as the key diagnostic tool often changed and/or confirmed the diagnosis in patients with an initial diagnosis of MINOCA, which led to the use of appropriate guideline-recommended treatment. True MI was confirmed in $38 \%$ of patients in our study group.

Cardiac magnetic resonance was safe in all patients, and a very high rate of diagnostic CMR results was obtained $(100 \%$ of patients). It also seems that CMR should be used more frequently in clinical practice, which could be facilitated by further studies in larger groups of patients.

\section{Conflict of interest: none declared}

\section{References}

1. Thygesen K, Alpert J, Jaffe A, et al. Fourth universal definition of myocardial infarction (2018). Eur Heart J. 2018; 40(3): 237-269, doi: 10.1093/eurheartj/ehy462.

2. Agewall S, Beltrame JF, Reynolds HR, et al. ESC working group position paper on myocardial infarction with non-obstructive coronary arteries. Eur Heart J. 2017; 38(3): 143-153, doi: 10.1093/eurheartj/ehw149, indexed in Pubmed: 28158518.

3. Opolski M, Spiewak M, Marczak M, et al. Mechanisms of myocardial infarction in patients with nonobstructive coronary artery disease. JACC: Cardiovasc Imaging. 2018 [Epub ahead of print], doi: 10.1016/j.jcmg.2018.08.022, indexed in Pubmed: 30343070.

4. Friedrich MG, Sechtem U, Schulz-Menger J, et al. Cardiovascular magnetic resonance in myocarditis: A JACC White Paper. J Am Coll Cardiol. 2009; 53(17): 1475-1487, doi: 10.1016/j. jacc.2009.02.007, indexed in Pubmed: 19389557.

5. Scantlebury DC, Prasad A, Scantlebury DC, et al. Diagnosis of Takotsubo cardiomyopathy. Circ J. 2014; 78(9): 2129-2139, indexed in Pubmed: 25131525.

6. Ouldzein H, Elbaz M, Roncalli J, et al. Plaque rupture and morphological characteristics of the culprit lesion in acute coronary syndromes without significant angiographic lesion: analysis by intravascular ultrasound. Ann Cardiol Angeiol (Paris). 2012; 61(1): 20-26, doi: 10.1016/j.ancard.2011.07.011, indexed in Pubmed: 21903196.

7. Gościniak P, Baron T, Józwa R, et al. MINOCA in cardiological standards 2018 and the use of magnetic resonance. Warszawa; Medical Tribune: 2018.

Cite this article as: Gościniak P, Baron T, Józwa R, et al. The tip of the iceberg: cardiac magnetic resonance imaging findings in patients with myocardial infarction with non-obstructive coronary arteries: preliminary data from the Polish single-centre registry. Kardiol Pol. 2019; 78(3): 389-392, doi: 10.5603/KP.a2019.0001. 